\title{
DISTRIBUCION DE LAS ESPECIES DEL GENERO HYDROPSYCHE (O. TRICHOPTERA, HYDROPSYCHIDAE) EN LA CUENCA DEL ALTO TAJO (GUADALAJARA)
}

\author{
J.M. Herranz y D. García de Jalón \\ Departamento de Zoología y Entomología E T S de Ingenieros de Montes Madrid
}

Palabras clave Trichoptera, Tajo river (Spain)

\begin{abstract}
DISTRIBUTION OF THE ESPECIES OF HYDROPSYCHE IN THE UPPER TAJO RIVER BASIN

During 1979, 1980 and 1981,302 samples of benthic macroinvertebrates were taken in 27 sampling points, spread along the upper part of the river Tajo Basin (Spain), between 730 and 1,560 m. of altitude.

Six different species of the genus Hydropsyche Pictet 1834 (Trichoptera, Insecta) were found: H. contubernalis, H. cf. dinarica, $H$. instabilis, $H$. pictetorum, $H$. siltalai and $H$. sp. The distribution of these species, according to the altitude, distance to the sources and several physico-chemical parameters, is studied.
\end{abstract}

\section{INTRODUCCION}

Las larvas de Hydropsyche son reófilas y habitan principalmente bajo la superficie de los sustratos pedregosos o entre los tallos de las macrofitas donde, bajo ciertas condiciones de corriente, tejen redes por medio de las cuales capturan la materia orgánica en suspensión que arrastran las aguas. De esta forma retardan el movimiento dominante de esta materia orgánica hacia aguas abajo, reteniendola y alterándola a la vez, por lo que desempeñan un papel importante en el funcionamiento del ecosistema fluvial (Merrit \& Wallace, 1981).

En la Cuenca del Alto Tajo hemos capturado diversas especies del género Hydropsyche cuya distribución y características mesológicas se analizan en el presente trabajo.

\section{DESCRIPCION DE LA ZONA DE ESTUDIO}

La Cuenca del Alto Tajo comprende el cuadrante sureste de la provincia de Guadalajara, parte norte de la provincia de Cuenca y una pequeña porción de la Sierra de Albarracin, en la provincia de Teruel. Limita, al norte con la cuenca del Ebro; al este, con la del Guadalviar; y al sur, con la del Júcar.

La zona objeto de estudio incluye la cuenca del Tajo desde su nacimientoen la Sierra de Albarracín hasta la cola del embalse de Entrepeñas, en las proximidades de Trillo, habiendo realizado muestreos en el tramo indicado del río Tajo y en los ríos Hoz-Seca, Cabrillas y Gallo a lo largo de todo su recorrido (Figura 1).

Los ríos discurren entre terrenos de naturaleza geológica muy diferente calizas, margas, pizarras, cuarcitas, conglomerados, areniscas y arcillas siendo los terrenos calizos los mejor representados lo que determina la existencia de frecuentes filtraciones de aguas que luego afloran bruscamente.

Floristicamente, la cuenca del Alto Tajo pertenece al dominio mediterraneo con transición al eurosiberiano. Los enclaves de flora eurosiberiana se deben a que la altitud da lugar a una mayor humedad ambiental y aportación hídrica por precipitaciones. Las principales especies arbóreas son: Pinus sylvestris, Pinus nigra, Pinus pinaster, Pinus 


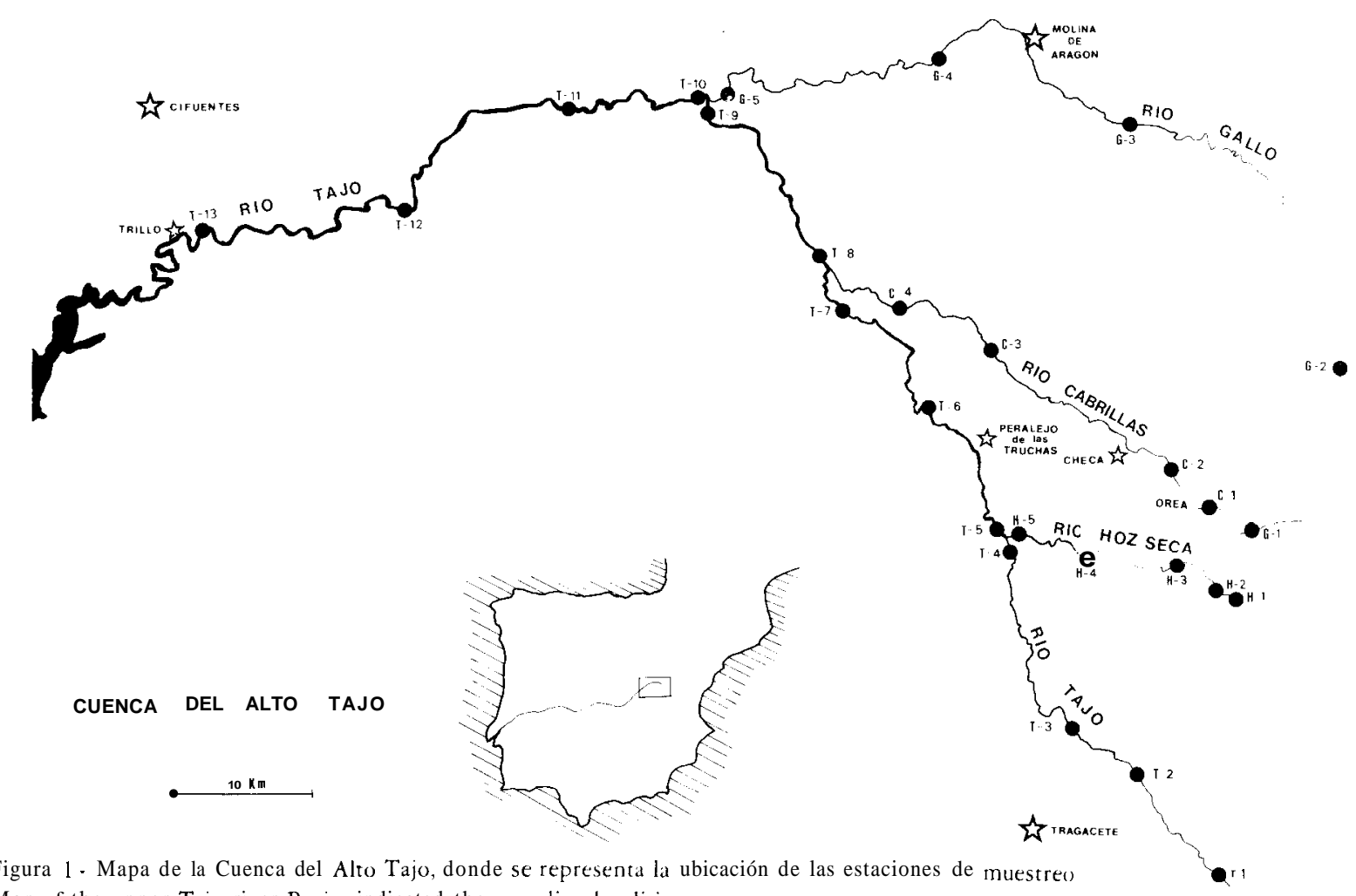

Figura 1. Mapa de la Cuenca del Alto Tajo, donde se representa la ubicación de las estaciones de muestreo Map of the upper Tajo river Basin, indicated the sampling localities.

balepensis, Quercus ilex, Quercus faginea, y Juniperus thurifera. Entre la vegetación ripícola predominan Salix sp y Populus nigra.

Las precipitaciones anuales oscilan entre 600 y 800 $\mathrm{m} m$ en las vertientes del río Tajo y entre 500 y 600 $\mathrm{mm}$ anuales en las zonas más próximas al río Gallo. Las temperaturas medias anuales varían entre $7^{\circ} \mathrm{y}$ $12^{\circ} \mathrm{C}$.

\section{METODOLOGIA}

Hemos elegido 27 estaciones de muestreo distribuidas 13 en el río Tajo, $S$ en el Hoz-Seca, 4 en el Cabrillas y $\mathrm{S}$ en el Gallo.

En la ubicación de las estaciones de muestreo se han considerado como criterios de elección la pendiente, la naturaleza del sustrato que forma el lecho del río, el tipo de vegetación de las márgepes y terrenos próximos, la confluencia de dos ríos, los aportes orgánicos, la posible sequía temporal de un tramo del río y la facilidad de acceso a las mismas.

En el mapa de la Figura 1 se refleja la situación de las estaciones de muestreo. Las estaciones Gallo-2 y Hoz-Seca-4 son representativas de tramos de río que solo llevan agua en épocas de grandes riadas. En la estación Gallo-2 no hemos tenido ocasión de realizar nigún muestreo, al hallarse completamente seca todas las veces que hemos visitado la zona.

Los muestreos de fauna se han realizado colectando directamente, con red tipo surber con una malla de 0,7 $\mathrm{mm}$ de luz, e indirectamente, por medio de sustratos artificiales, durante los años 1979, 1980, 1981.

En los muestreos directos se han realizado dos réplicas o extracciones, en cada una de las cuales se han recolectado los organismos existentes en una superficie rectangular de $25 \times 30 \mathrm{~cm}$, elegida de forma que captara la heterogeneidad de los hábitats existentes. Los sustratos artificiales, consistentes la mayoría de ellos en ladrillos de cerámica rellenos de gravas, han sido sometidos a periodos de colonización oscilando entre 1 y 10 semanas.

En total se han realizado 105 muestreos directos y 197 por medio de sustratos artificiales.

\section{RESULTADOS}

Analisis esporádicos de las características físicoquímicas de las aguas de la zona estudiada, realizados 
Tabla I.- Distribución de las especies de Hydropsyche en las distintas estaciones de muestreo de la cuenca del Alto Tajo. En los casos de presencia, cada especie está caracterizada por dos números: el de arriba indica el número de muestreos que ha sido colectada en la estación de muestreo y el segundo, el número total de ejemplares capturados.

Distribution of the species of Hydropryche in the upper Tajo Basin. Each species is characterized by two numbers: the upperone means the number of samples in which it has been caught and the other one, the number of specimens collected

\begin{tabular}{|c|c|c|c|c|c|c|c|c|c|c|c|c|c|c|c|c|c|c|c|c|c|c|c|c|c|c|}
\hline Río & \multicolumn{14}{|c|}{ TAJO } & \multicolumn{3}{|c|}{ HOZ-SECA } & \multicolumn{5}{|c|}{ CABRILLAS } & \multicolumn{4}{|c|}{ GALLO } \\
\hline Estación de muestre" & $\mathrm{T}-1$ & $\mathrm{~T}-2$ & $\mathrm{~T}-3$ & $\mathrm{~T}-4$ & $\mathrm{~T}-5$ & T-6 & T-7 & $\mathrm{T}-8$ & T-9 & $\mathrm{T}-10$ & $T-11$ & $\mathrm{~T}-12$ & $T-13$ & $H-1$ & $\mathrm{H}-2$ & $H-3$ & $H-4$ & $Y-<C$ & -1 & $C-?$ & $c-3 c$ & C-4 & $G-1$ & $C-3$ & $G-4$ & $c-5$ \\
\hline Número de muestreos & 4 & 7 & 15 & 6 & 2 & 21 & 20 & 18 & 17 & 21 & 7 & 15 & 7 & 4 & 20 & 15 & 1 & 6 & 3 & 12 & 21 & 13 & 7 & 5 & 21 & 14 \\
\hline H. cf. dinarica & - & 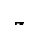 & $\begin{array}{l}4 \\
21\end{array}$ & $\begin{array}{l}1 \\
1\end{array}$ & - & $\begin{array}{l}6 \\
20\end{array}$ & $\begin{array}{l}6 \\
20\end{array}$ & $\begin{array}{l}3 \\
4\end{array}$ & $\begin{array}{l}1 \\
1\end{array}$ & - & - & $\pi$ & - & - & $\begin{array}{l}6 \\
16\end{array}$ & $\begin{array}{l}1 \\
4\end{array}$ & - & - & $\begin{array}{l}2 \\
11\end{array}$ & $\begin{array}{l}8 \\
22\end{array}$ & $\begin{array}{c}3 \\
18\end{array}$ & $\begin{array}{l}7 \\
10\end{array}$ & $\begin{array}{l}5 \\
21\end{array}$ & - & $\begin{array}{l}2 \\
5\end{array}$ & \\
\hline H. instabilis & $=$ & - & $\stackrel{4}{10}$ & - & - & $\frac{12}{36}$ & $\begin{array}{r}12 \\
102\end{array}$ & $\begin{array}{l}30 \\
20\end{array}$ & $\frac{1}{1}$ & - & - & - & & - & in & ? & - & in & $\begin{array}{l}3 \\
19\end{array}$ & $\begin{array}{l}6 \\
17\end{array}$ & $\begin{array}{l}11 \\
87\end{array}$ & $\begin{array}{l}5 \\
16\end{array}$ & $\begin{array}{l}2 \\
7\end{array}$ & - & $\begin{array}{l}4 \\
24\end{array}$ & $\begin{array}{l}1 \\
1\end{array}$ \\
\hline H. pictetorum & - & $\begin{array}{l}1 \\
1\end{array}$ & $\begin{array}{l}4 \\
7\end{array}$ & - & - & $\begin{array}{l}13 \\
42\end{array}$ & $\begin{array}{l}13 \\
76\end{array}$ & $\begin{array}{l}11 \\
33\end{array}$ & $\begin{array}{l}8 \\
21\end{array}$ & $\begin{array}{r}15 \\
117\end{array}$ & $\begin{array}{l}2 \\
3\end{array}$ & $\begin{array}{l}3 \\
4\end{array}$ & $\begin{array}{l}1 \\
1\end{array}$ & - & $\begin{array}{l}7 \\
35\end{array}$ & $\begin{array}{l}5 \\
10\end{array}$ & - & - & $\begin{array}{l}2 \\
3\end{array}$ & $\begin{array}{l}1 \\
2\end{array}$ & $\begin{array}{l}7 \\
57\end{array}$ & $\begin{array}{l}8 \\
40\end{array}$ & $\begin{array}{l}1 \\
10\end{array}$ & $\begin{array}{l}4 \\
57\end{array}$ & $\begin{array}{r}17 \\
408\end{array}$ & $\begin{array}{l}\stackrel{9}{5} \\
50\end{array}$ \\
\hline H. siltalai & - & - & - & - & - & $=$ & - & - & - & - & - & - & $=$ & $\begin{array}{l}1 \\
1\end{array}$ & $\begin{array}{l}7 \\
21\end{array}$ & $\begin{array}{l}5 \\
6\end{array}$ & - & - & $\begin{array}{l}7 \\
10\end{array}$ & $\begin{array}{l}9 \\
23\end{array}$ & $\begin{array}{l}5 \\
8\end{array}$ & & $\begin{array}{l}6 \\
34\end{array}$ & $\begin{array}{l}3 \\
6\end{array}$ & $\begin{array}{r}11 \\
485\end{array}$ & - \\
\hline Hydropsyche sp & - & - & - & - & - & - & 8 & 3 & 5 & 91 & $\xi_{1}$ & $\stackrel{6}{33}$ & $\frac{1}{1}$ & & - & - & - & - & - & - & - & - & - & - & $\begin{array}{l}2 \\
2\end{array}$ & $\begin{array}{l}2 \\
14\end{array}$ \\
\hline
\end{tabular}

con un laboratorio portátil Hach, dieron como resultado valores de oxígeno disuelto altos, superiores al $100 \%$ de saturación, pequeños contenidos de nitratos y fosfatos, a excepción de las estaciones de muestreo Gallo-4, Gallo-5 y Tajo-10 donde se midieron los siguientes:

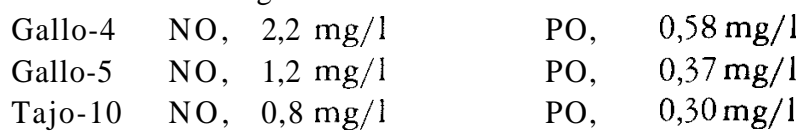

Entre los organismos capturados hemos identificado todas en estado larvario las siguientes especies:

Hydropsyche contubernalis Mac Lachlan, 1865

Hydropsyche cf, dinarica Marinkovic, 1979

Hydropsyche instabilis Curtis, 1834

Hydropsyche pictetorum Botosaneanu \& Schmid, 1973

Hydropsyche siltalai Dohler, 1963

Hidropsyche sp.

Los ejemplares de Hydropryche sp no han podido ser determinados a nivel específico, pero son ciertamente una especie diferente a las anteriores.

En la tabla I se expresa la presencia/ausencia de las distintas especies de Hydropsyche e n las estaciones de muestreo. En dicha tabla figura el número total de muestreos realizados en cada estación, el número de muestreos en que ha sido capturada cada especie en las distintas estaciones y el número total de individuos capturados.

En ia Tabla 2 se expresan las variaciones de los parámetros físico-quimicos entre las estaciones en que habitan las diferentes especies de Hydropsyche en la zona estudiada.

\section{DISCUSION}

Hydropsyche cf dinarica e Hydropsyche instabilis habitan bajo condiciones ecológicas muy semejantes en la zona estudiada, como queda reflejado en la Tabla 2. Presentan un amplio espectro altitudinal y viven tanto en aguas silíceas como calizas. Para Verneaux y Faessel (1976) Hydropryche instabilis es una especie rhithrobionte. Decamps (1967) la cita en los Pirineos Franceses habitando en ríos que tienen aportes nutritivos importantes debidos ya sea a las partículas 
Tabla II.- Variaciones de algunos de los parámetros estudiados en las estaciones de muestreo en que habitan las diferentes espcies de Hydropsyche en la cuenca del Alto Tajo.

Variations of some characteristics the sampling localiries where species of Hydropsyche were found.

\begin{tabular}{|c|c|c|c|c|c|}
\hline & $\begin{array}{l}\text { Altitud } \\
(\mathrm{m})\end{array}$ & $\mathrm{pH}$ & $\begin{array}{l}\text { Conductividad } \\
\text { (umhos / cm.) }\end{array}$ & $\begin{array}{l}\text { Alcalinidad } \\
(\mathrm{mg} / 1 .)\end{array}$ & $\begin{array}{c}\text { Dureza } \\
(\mathrm{mg} / \mathrm{l})\end{array}$ \\
\hline Hydropsyche cf. dinarica & $890-1520$ & $7,4-8,6$ & $135 \cdot 1052$ & $28-238$ & $49 \cdot 434$ \\
\hline Hydropsyche instabilis & $890=1520$ & $7,4-8,7$ & $135-1502$ & $28 \cdot 238$ & $49 \cdot 414$ \\
\hline Hydropsyche picterorum & $730-1520$ & $7,4-8,7$ & $115 \cdot 1052$ & $28+238$ & $49-434$ \\
\hline Hydropsyche siltalai & $1010-1560$ & $6,7 \cdot 8,6$ & $87 \cdot 1052$ & $14-238$ & $21-434$ \\
\hline Hydropsycbe contubernalis & $710-830$ & $8,2-8,4$ & $662 \cdot 679$ & $196-224$ & $280-301$ \\
\hline Hydropsyche sp & $710-1020$ & $8,0-8,7$ & $605 \cdot 1052$ & $196 \cdot 238$ & $245-420$ \\
\hline
\end{tabular}

arrastradas por las aguas o a las acumulaciones de hojas muerta. La larva de $H$. cf dinarica ha sido descrita recientemente por García de Jalón (1983)

Hydrosyche pictetorum ha sidoencontrada por uno de nosotros (García de Jalón, 1982) en pequeños ríos calizos de la provincia de Guadalajara, estando presente tanto en tramos de gran pendiente como en otros donde el cauce se ensancha y las aguas se eutrofizan. En la zona objeto del preser'e estudio, esta especie participa también de un marcado carácter eurioico, habitando tanto en los tramos superiores de los ríos como en los tramos bajos, presentando una gran abundancia en la estación Tajo-10, inmediatamente aguas abajo de la llegada a este río de las aguas eutrofizadas del río Gallo. Las estaciones de muestreo en que ha sido capturado tienen grandes variaciones en altitud y en las características físicoquímicas de las aguas (Tabla II).

Hydropsyche sillalai ha sido colectada en las partes más altas de los ríos Hoz-Seca, Cabrillas y Gallo, entre 1010 y $1560 \mathrm{~m}$ de altitud; las características físicoquímicas de las aguas en que habita presentan grandes variaciones de unas estaciones a otras (Tabla 2), mostrando la especie un caracter eurioico señalado también por Verneaux y Faessel 1976. Según Giudicelli et al. 1980 se trata de una especie que prefiere los tramos superiores y medios de los cursos de agua, En Portugal es la especie del género más frecuente en pequeños ríos con mucha velocidad de corriente, en general en regiones montañosas (da Terra, 1981).

Hydropsyche contubernalis es característica del tramo del río Tajo situado entre 730 y $830 \mathrm{~m}$ de altitud , donde habita en aguas calcáreas, básicas y duras, Puig et al. 1981 citan a esta especie en el río Llobregat habitando en aguas calcáreas y duras, con ligera contaminación. En los grandes ríos europeos $H$. contubernalis sustituye progresivamente aguas abajo a H. pellucidula (Bournand et al., 1982) especie curiosamente ausente en el Alto Tajo.

Hydropsyche sp. vive en los ríos Tajo y Gallo, entre 730 y 1020 m de altitud. Habita en aguas de características físico-quimicas muy parecidas a las exigidas por Hydropsyche contubernalis, especie con la que se encuentra asociada frecuentemente.

Estos resultados permiten señalar a las especies $\mathrm{H}$. cf. dinarica e $H$. instabilis como caracteristicas de los tramos altos de la zona estudiada y a H.contubernalis e H. $s p$. como características de los más bajos, si bien, la relativa uniformidad ecológica de las aguas a que nos referimos no permite establecer una zonación clara de la misma con dichas especies.

\section{BIBLIOGRAFIA}

Bournaud, M.; Tachet, H. \& Perin, J.F. 1982. Les Hydropschidac (trichoptera) du Haut-Rhone entre Geneve et Lyon Annls Limnol 18(1): 61-80

Decamps, H. 1967. Ecologie des trichoptéres de la Vallée d'Aure (Haute Pyrenées) Amnls Limnol 3(3): 399-577.

Guiudicelli, J.; Dia, A. y Legier, P.1980. Erude hydrobiologique d'une riviére de region méditérranéenne, L'Argens Habitats hydruchimie, distribution de la faune benthique Bigdragen tot de Dierkunde. $50(2) 303-341$

García de Jalón, D. 1982.Contribución a la zoogeografía de los tricópteros españoles. SHILAP 10: 75078; 157-165;237-244

García de Jalón, D. 1983 Contribución al conocimiento de las larvas del género $H_{y} d r o p s y c h e$ ibéricas (trichoptera nnsecta). Actas I Congreso lber Entomol. 275-285

Merrit, R.W. y Wallace, J.B..1981. Insectos filtradores Investigación y Ciencia 57 275-285

Puig, A.; Bautista, I.; Tort, M.J. y Prat, N. 1981. les larves de trichoptéres de la riviére Llobregat Distribution longitudinale et relation avec la qualité de l'eau Proces. 3rd. Int Syinp Trich 303. 310 (j Moretti (ed) Junk

Verneax, J. y Faessel, B. 1976. Larves du genre Hidrotriche Taxonomie, donées biologiques et écologiques Annls. Limnol. $12(1) 7-16$

da Terra, L.S.W. 1981. Lista faunistica de tricopteros de Portugal Bol Soc Port rnt 12 